\title{
Aplicación de los modelos cinéticos Logístico Integrado y Gompertz Modificado para la producción de bioetanol en procesos fermentativos a partir de mucílago de cacao CCN-51
}

\author{
Jorge Delgado $^{1 *}$, Jaime Soler², José Ángel Peña ${ }^{2}$ \\ ${ }^{1}$ Facultad de Ciencias Químicas, Carrera de Ingeniería Química, Universidad Cuenca; Cuenca, Ecuador \\ ${ }^{2}$ Grupo de Catálisis, Separaciones Moleculares e Ingeniería de Reactores (CREG) \\ Instituto de Investigación en Ingeniería de Aragón (I3A) \\ Universidad Zaragoza, Mariano Esquillor 3, 50018, Zaragoza, Spain. \\ *e-mail: jorge.delgado@ucuenca.edu.ec
}

\section{Resumen}

El objetivo de este estudio es modelar la producción de bioetanol generado en la fermentación de mucílago de Cacao CCN - 51 con levadura del tipo Saccharomyces Cerevisiae y la aplicación de los modelos: Logístico Integrado y Gompertz modificado. [1]

En Ecuador no se ha valorado el mucílago de cacao, que es un residuo que presenta un alto potencial energético en la producción de bioetanol como aditivo de la gasolina.

La fermentación discontinua se desarrolló en un biorreactor de tipo batch bajo diferentes condiciones de $\mathrm{pH}$, temperatura y concentración de levadura. Durante la reacción se evaluó, la cantidad de biomasa y bioetanol generado, mediante los métodos de peso seco por liofilización y microdifusión respectivamente.

El resultado de la concentración final de bioetanol fue de $25,41 \mathrm{~g} / \mathrm{L}$ a una temperatura de $35^{\circ} \mathrm{C}$, $\mathrm{pH}$ de 4 y concentración de levadura de $3 \mathrm{~g} / \mathrm{L}$. A partir de los resultados, se utilizó el modelo logístico para describir el crecimiento de la levadura y el modelo Gompertz modificado para modelar la producción de bioetanol, ajustando ambos modelos con coeficientes de determinación $\left(R^{2}\right)$ superiores a 0,9.

\section{Introducción}

La producción de biocombustibles ha despertado gran interés, en los campos económico, social, ambiental, institucional y político. En primer lugar, los altos precios del petróleo hacen que los países que no son autosuficientes en el suministro de combustibles fósiles, destinen una parte importante de sus divisas en la importación de hidrocarburos. En el orden social, la necesidad de diversificar las fuentes de energía y de investigar los recursos locales ha repercutido en una mayor generación de empleo, pues ha sido necesario contratar nueva mano de obra para esta actividad productiva. En el aspecto ambiental, los biocombustibles son menos contaminantes y tienen menor impacto sobre el cambio climático que los combustibles fósiles.

Finalmente, esta posibilidad de obtener una fuente de energía secundaria va en la corriente mundial de reemplazo gradual de los combustibles fósiles y la generación de nuevas áreas de conocimiento en temas energéticos, que pueden ser utilizados en un futuro como fuente primaria para la producción del bioetanol como vector energético [2]. Los combustibles fósiles, tales como el petróleo, gas natural y carbón, como fuentes de energía no renovable, están presentando señales de agotamiento, estimándose que a mediano plazo ya no será posible cubrir la demanda mundial de energía. Además, a ésta situación se suma la contaminación ambiental y el calentamiento global, provocados en gran medida por el uso de este tipo de combustibles. [3]

Por lo antes expuesto, el mucílago de cacao que en la actualidad es un residuo sin aprovechamiento útil y rentable, resulta ser de alto potencial energético en la producción de bioetanol para uso como aditivo en gasolinas.

\section{Experimental}

El residuo mucilaginoso de cacao CCN-51 corresponde a la zona geográfica 6 del Ecuador, en el cantón La Troncal, punto de georreferencia: $2^{\circ} 29^{\prime} 25^{\prime}$ 'S y $79^{\circ} 19^{\prime} 3$ ’’O. Esta variedad se utilizó debido a su mayor productividad en el Ecuador.

Revista “Jornada de Jóvenes Investigadores del I3A”, vol. 7 (Actas de la VIII Jornada de Jóvenes Investigadores del I3A - 6 de junio de 2019). ISSN 2341-4790. 
La fermentación alcohólica se llevó a cabo en un biorreactor homogéneo de tipo batch de marca Biotron $G X$ con una agitación constante de $250 \mathrm{rpm}$. La experimentación transcurrió entre 30 y 96 horas dependiendo de los parámetros de operación y utilizando levadura del tipo Saccharomyces Cerevisiae.

La cuantificación de biomasa se realizó mediante el método analítico peso seco. Este método consistió en la toma de $5 \mathrm{~mL}$ de muestras mucilaginosas del fermentador, seguido de una centrifugación a 4000 rpm durante $10 \mathrm{~min}$. Las muestras fueron almacenadas en nitrógeno líquido a $-190{ }^{\circ} \mathrm{C}$ para evitar reacciones de degradación. Cuando concluyó el proceso experimental, las muestras fueron liofilizadas en un liofilizador FT 33 marca Armfield, permaneciendo en el mismo por 48 horas. Las primeras 24 horas se realizó la congelación y en las 24 horas siguientes se realizó el secado. Finalmente se realizó el pesaje de las muestras liofilizadas y los cálculos respectivos. [4]

La cuantificación del bioetanol durante la experimentación se realizó por el método de Winnick o microdifusión [5], para lo cual previamente, se centrifugaron las muestras durante 15 minutos a $4000 \mathrm{rpm}$ y se extrajo $1 \mathrm{~mL}$ de sobrenadante, mismo que se aforó a $100 \mathrm{~mL}$ con agua destilada.

La ecuación logística integrada se utilizó para modelar en el proceso de fermentación, la tasa de crecimiento específico máximo $\left(\mu_{\max }\right)$. La ecuación de Gompertz modificado se utilizó para modelar el proceso de fermentación, y determinar la velocidad máxima de producción de bioetanol (rpm). Los ajustes se realizaron empleando el método de mínimos cuadrados no lineales de Marquardt en MATLAB.

\section{Resultados y discusión}

El diseño factorial (no mostrado), permitió establecer las condiciones de operación que mayor producción de bioetanol generaron (Tabla 1), siendo estas la temperatura de $35^{\circ} \mathrm{C}, \mathrm{pH}$ de 4 y concentración de levadura de $3 \mathrm{~g} / \mathrm{L}$. Esto ocurrió en un tiempo de fermentación de 36 horas, tiempo en el cual, la concentración máxima experimental de bioetanol fue de 25,41 g/L y la calculada de 24,76 g/L con un rpm calculado de 1,13 g/(L.h), y la tasa de crecimiento específico máximo $\left(\mu_{\max }\right)$ calculada fue $0,39 \mathrm{~h}^{-1}$.

Los coeficientes de determinación obtenidos a partir de los datos experimentales de biomasa y bioetanol con los modelos Logístico Integrado (Figura 1) y Gompertz modificado (Figura 2), presentan valores de $\mathrm{R}^{2}$ superiores a 0,9 , lo que refleja el buen ajuste de los modelos.

Tabla 1. Resultados de la concentración de etanol experimentales y calculados mediamente la ecuación de Gompertz modificado del experimento con mayor producción de etanol

\begin{tabular}{ccc}
\hline Tiempo (h) & $\begin{array}{c}\text { Concentración de } \\
\text { etanol (g/L) } \\
\text { experimentales }\end{array}$ & $\begin{array}{c}\text { Concentración de } \\
\text { etanol (g/L) } \\
\text { calculado }\end{array}$ \\
\hline 0 & 0 & 0,77 \\
6 & 7,19 & 4,74 \\
12 & 9,11 & 11,30 \\
18 & 16,51 & 17,11 \\
24 & 21,10 & 20,85 \\
30 & 24,45 & 22,92 \\
36 & 25,41 & 23,98 \\
42 & 25,39 & 24,51 \\
48 & 25,40 & 24,76 \\
\hline
\end{tabular}

\section{Conclusiones}

El mucílago de cacao CCN-51, residuo de la creciente industria cacaotera, representa una materia prima con un gran potencial para la obtención de bioetanol, puesto que contienen azúcares fermentables y se encuentra de manera abundante en el Ecuador.

La aplicación de un diseño de experimentos, permitió establecer las condiciones de temperatura, pH y concentración de levadura con los cuales se generó la mayor producción de biomasa y bioetanol.

Este estudio utilizó modelos cinéticos empíricos como son el Logístico Integrado y el Gompertz modificado, que mostraron un buen ajuste con los datos experimentales obtenidos y presentan aceptabilidad para predecir y controlar sistemas fermentativos con mucílago de cacao CCN-51. 


\section{Referencias}

[1]. PHUKOETPHIM, N., SALAKKAM, A., LAOPAIBOON, P. and LAOPAIBOON, L. Kinetic models for batch ethanol production from sweet sorghum juice under normal and high gravity fermentations: Logistic and modified Gompertz models. Journal of Biotechnology, 243, 69-75. 2017 doi: 10.1016/j.jbiotec.2016.12.012 .

[2]. ARIYAJAROENWONG, P., LAOPAIBOON, P., SALAKKAM, A., SRINOPHAKUN, P. and LAOPAIBOON, L. Kinetic models for batch and continuous ethanol fermentation from sweet sorghum juice by yeast immobilized on sweet sorghum stalks, Journal of Taiwan Institute of Chemical Engineers, 66, 210-216, 2016.

[3]. HÖÖK, M., and TANG, X. Depletion of fossil fuels and anthropogenic climate change-A review, Energy Policy, 52, 797-809, 2013.

[4]. GARCÍA CUBERO, R. "Producción de biomasa de microalgas rica en carbohidratos acoplada a la eliminación fotosintética de $\mathrm{CO}_{2}$ " Tesis Doctoral. Universidad de Sevilla, 2014.

[5]. AUGUSTO, J. y MORA, H. Producción de etanol a partir de glicerina por vía fermentativa con Saccharomyces cerevisiae y simulación del proceso a escala industrial. Tesis de maestría. Universidad de Manizales, Colombia, 2015.

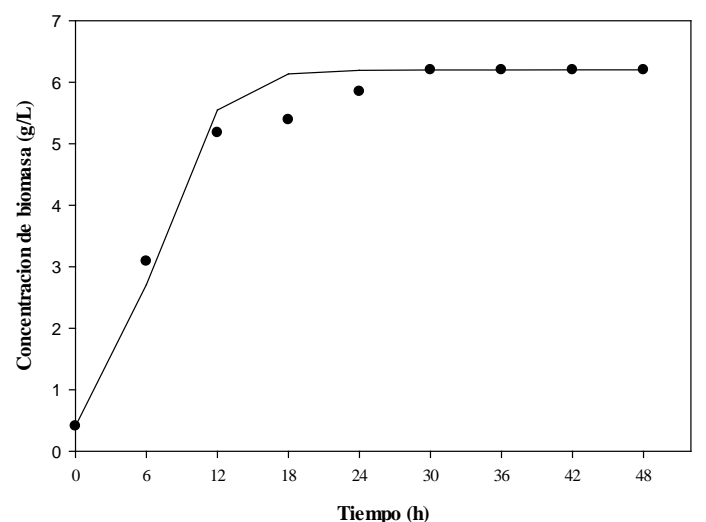

Figura 1. Curva de producción de biomasa. Símbolos: círculos cerrados, datos experimentales; línea continua, curva ajustada.

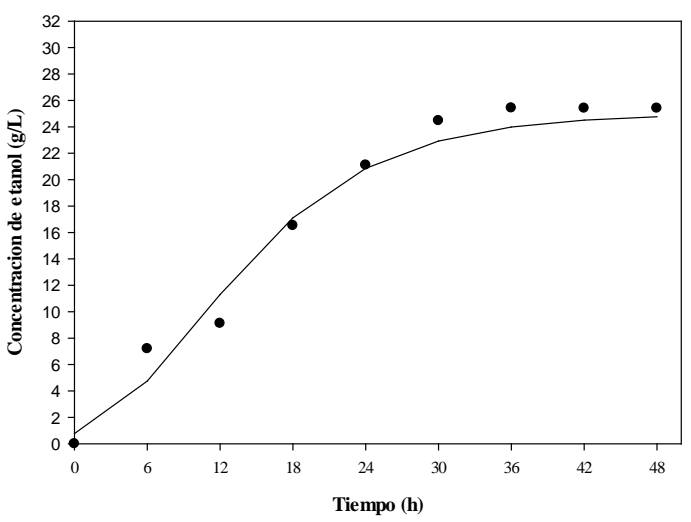

Figura 2. Comparación de la producción de bioetanol: Datos empíricos (símbolos) y modelo de Gompertz modificado (curva). 